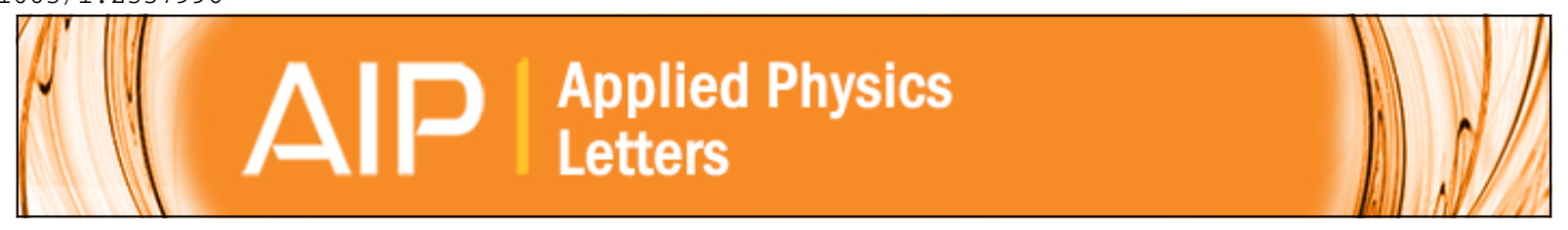

\title{
Giant magnetoelectric effect in Metglas/polyvinylidene-fluoride laminates
}

Junyi Zhai, Shuxiang Dong, Zengping Xing, Jiefang Li, and D. Viehland

Citation: Applied Physics Letters 89, 083507 (2006); doi: 10.1063/1.2337996

View online: http://dx.doi.org/10.1063/1.2337996

View Table of Contents: http://scitation.aip.org/content/aip/journal/apl/89/8?ver=pdfcov

Published by the AIP Publishing

\section{Over 700 papers \&}

presentations on

multiphysics simulation visw now

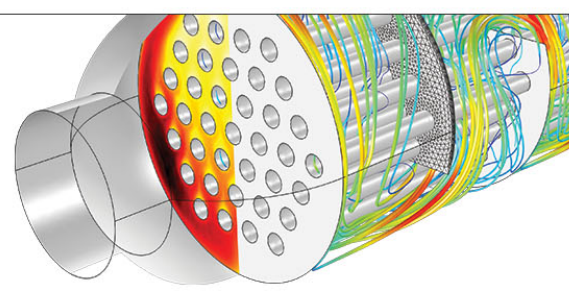




\title{
Giant magnetoelectric effect in Metglas/polyvinylidene-fluoride laminates
}

\author{
Junyi Zhai, ${ }^{a}$ Shuxiang Dong, Zengping Xing, Jiefang Li, and D. Viehland \\ Department of Materials Science and Engineering, Virginia Tech, Blacksburg, Virginia 24061
}

(Received 5 June 2006; accepted 29 June 2006; published online 22 August 2006)

\begin{abstract}
Here, the authors report thin $(<100 \mu \mathrm{m})$ and flexible magnetoelectric $(\mathrm{ME})$ composites consisting of Metglas (high- $\mu$ magnetostriction) and polyvinylidene-fluoride (piezopolymer) layers laminated together. Both unimorph and three-layer configurations have been studied. The authors find that these ME laminates (i) require dc magnetic biases as low as 8 Oe to (ii) induce giant ME voltage coefficients of $7.2 \mathrm{~V} / \mathrm{cm}$ Oe at low frequencies, and up to $310 \mathrm{~V} / \mathrm{cm}$ Oe under resonant drive. (C) 2006 American Institute of Physics. [DOI: 10.1063/1.2337996]
\end{abstract}

The magnetoelectric (ME) effect is defined as an induced dielectric polarization under an applied magnetic field $(H)$ and/or an induced magnetization under an external electric field $(E)$. The $\mathrm{ME}$ effect was first observed in $\mathrm{Cr}_{2} \mathrm{O}_{3}$ (Refs. 1 and 2) over 40 years ago, as previously summarized in Ref. 3. Recently, single phase ME materials have undergone a Renaissance, ${ }^{4-6}$ however, they exhibit only weak ME effects at quite low temperatures, and thus are not suitable for practical applications. Alternatively, composites $^{7-10}$ of magnetostrictive ferrites and piezoelectric $\mathrm{Pb}(\mathrm{Zr}, \mathrm{Ti}) \mathrm{O}_{3}$ (or PZT) layers laminated together have been found to have ME voltage coefficients of $V_{\mathrm{ME}}=0.05-0.4 \mathrm{~V} / \mathrm{cm}$ Oe. The highest values of $V_{\mathrm{ME}}$ are found for magnetostrictive Terfenol-D (Ref. 11) or Fe-Ga (Ref. 12) layers laminated with piezoelectric $\mathrm{Pb}\left(\mathrm{Mg}_{1 / 3} \mathrm{Nb}_{2 / 3}\right) \mathrm{O}_{3}-\mathrm{PbTiO}_{3}$ (PMN-PT) ones: values as high as $V_{\mathrm{ME}}=2.2 \mathrm{~V} / \mathrm{cm}$ Oe have been reported. Net forming of more complex shapes has been achieved by addition of a polyvinylidene fluoride (PVDF) piezopolymer phase to Terfenol-D/PZT composites: ${ }^{13,14}$ the high resistance of PVDF also reduces the eddy current losses of the composite. Prior investigations in 2001 (Ref. 15) predicted a giant ME effect in Terfenol-D/PVDF laminate and particulate composites. In 2002, Mori and Wuttig ${ }^{16}$ then reported the ME properties of such a Terfenol-/PVDF laminate composite.

A limitation of previously reported ME laminates is a low permeability in the magnetostrictive layers. This limitation, in conjunction with the demagnetization field, has required relatively high dc magnetic biases of $H_{\mathrm{dc}, \mathrm{opt}}$ $\approx 400$ Oe applied by permanent magnets in order to achieve a maximum effective piezomagnetic coefficient. Lowering of this required $H_{\mathrm{dc} \text {,opt }}$ by use of an alternative magnetostrictive phase is an important goal, which would enable laminate miniaturization for applications in magnetic sensing ${ }^{17}$ and transducer. ${ }^{18}$ Here, we report new ME laminates made of small strain but high magnetic permeability $\left(\mu_{r}>40000\right)$ Metglas $^{19}$ layer(s) laminated together with piezopolymer PVDF ones (with a high piezoelectric voltage constant). Such laminates have giant ME voltages under dramatically lower magnetic biases than previously reported for other material layer couples.

Iron-based Metglas 2605 SA1 or CO have compositions of $\mathrm{FeBSiC}$ or FeSiCo, both with magnetostrictions of 27-45 ppm. The thicknesses of our Metglas 2605 SA1 and CO layers were 25 and $23.5 \mu \mathrm{m}$, respectively. These thin

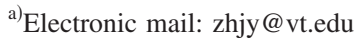

layers were obtained from Metglas, Inc. (Conway, SC). Without annealing, Metglas has a high dc permeability $>40000$ for a FeBSiC alloy and >120000 for a FeSiCo alloy: after annealing, their permeability are increased; however, their magnetoelastic properties are decreased. The piezoelectric layers we used were PVDF thin films of thickness of $28 \mu \mathrm{m}$. The Metglas layer and PVDF layers were glued together using an epoxy. Figure 1(a) shows a photo of a Metglas/PVDF laminate: unlike previous ME laminates, this Metglas/PVDF one was very thin, flexible, and capable of being bent (shown in the figure). Figures 1(b) and 1(c) show Metglas/PVDF laminate configurations: (b) is a unimorph consisting of single layers of Metglas $2605 \mathrm{CO}$ and PVDF that were epoxied together, whereas (c) is a sandwich structure, made of two layers of Metglas 2605 SA1 and a single PVDF layer. In both configurations, the Metglas layer(s) was longitudinally magnetized, whereas the piezoelectric ones were transversely poled. During the ME measurement, dc $\left(H_{\mathrm{dc}}\right)$ and ac $\left(H_{\mathrm{ac}}\right)$ magnetic fields were applied along the length of the laminates. An electromagnet was used to provide $H_{\mathrm{dc}}$ and a Helmholtz coil was used to generate $H_{\mathrm{ac}}$ $=1$ Oe. A lock-in amplifier (SR850) generated a controllable input current to the Helmholtz coil, and subsequently to measure the output voltage and phase from the PVDF film.

Figure 2(a) shows the dependence of $V_{\mathrm{ME}}$ on $H_{\mathrm{dc}}$ for our three-layer Metglas/PVDF laminate, measured at $1 \mathrm{kHz}$. The

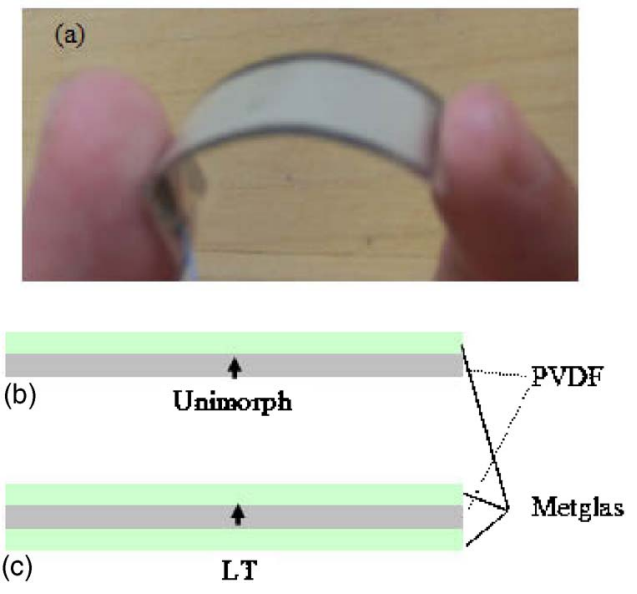

FIG. 1. (Color online) (a) Photograph of a Metglas/PVDF unimorph laminate and the structure of (b) the unimorph configuration, and (c) the threelayer LT sandwich configuration. In both configurations, the Metglas layer(s) was longitudinally magnetized, whereas the piezoelectric ones were transversely poled. 

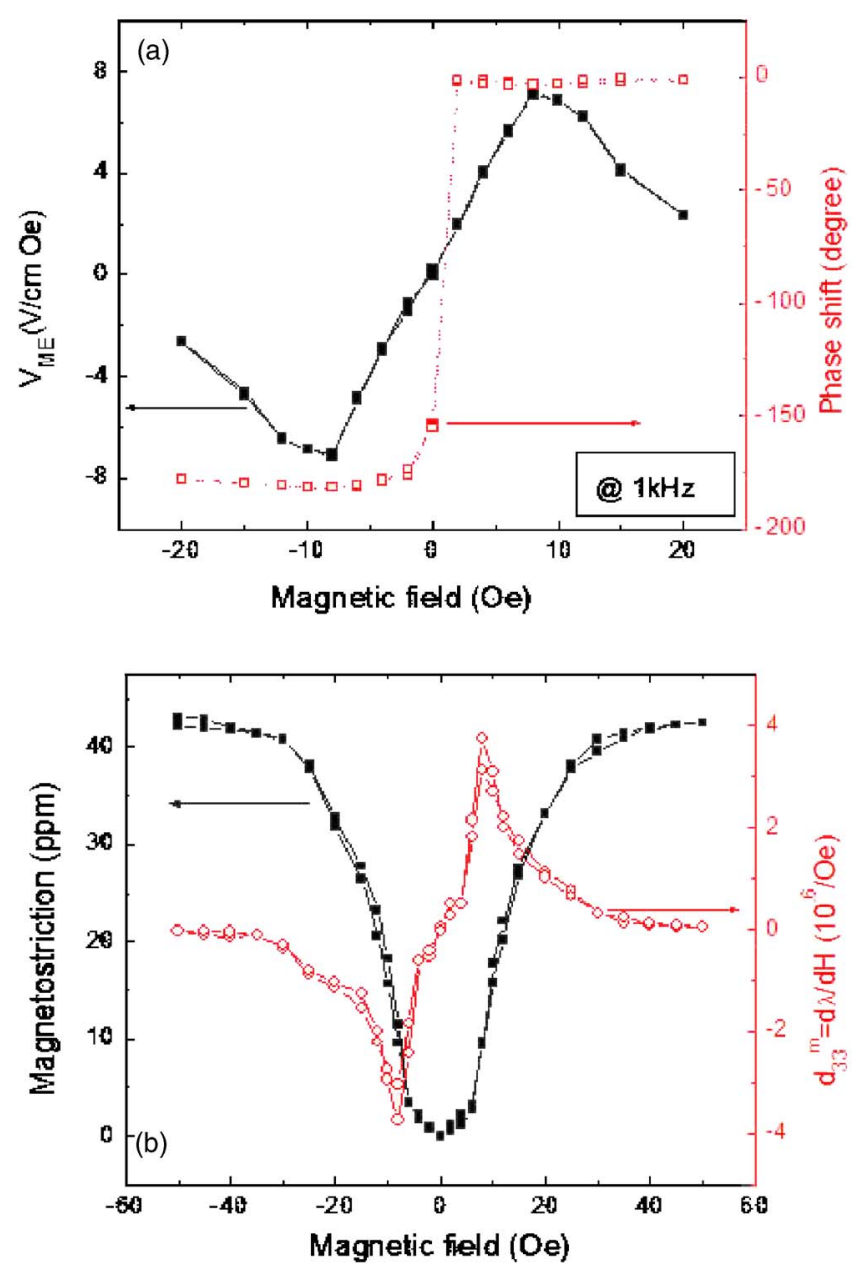

FIG. 2. (Color online) (a) dc magnetic field dependence of the ME voltage coefficient and phase for a Metglas/PVDF three-layer laminate, measured at $1 \mathrm{kHz}$ and $H_{\mathrm{ac}}=1 \mathrm{Oe}$; (b) magnetostriction (closed square) and piezomagnetic coefficient (open dot) for a Metglas (2605 SA1) layer in the LT mode.

maximum value of $V_{\mathrm{ME}}$ was $7.2 \mathrm{~V} / \mathrm{cm}$ Oe under $H_{\mathrm{dc}}=8$ Oe: this is three times greater than the largest value previously reported for three-layer Terfenol-D/PMN-PT longitudinal magnetization (LT) laminates. ${ }^{10}$ However, it is important to note that $H_{\mathrm{dc}}$ was only $8 \mathrm{Oe}$, which is $\sim 1 / 50$ th of that needed for the maximum ME effect in Terfenol-D/PMN-PT laminates. According to magnetoelectric equivalent circuit method, ${ }^{11,20}$ the ME voltage coefficient $V_{\mathrm{ME}}$ for LT laminates can be derived as ${ }^{21}$

$$
V_{\mathrm{ME}}^{\mathrm{LT}}=\frac{\left|d E_{1}\right|}{\left|d H_{3}\right|}=\frac{n d_{33, m} d_{31, p}}{n \varepsilon_{33}^{S} s_{11}^{E}+(1-n) s_{33}^{H}\left(\varepsilon_{33}^{S}+d_{31, p}^{2} / s_{11}^{E}\right)},
$$

where $n$ is the magnetic phase thickness ratio, $s_{11}^{E}$ and $s_{33}^{H}$ are the elastic compliances of the piezoelectric and magnetostrictive layers, respectively, $\varepsilon_{33}^{S}$ is the dielectric constant of the piezoelectric material at constant strain, and $d_{33, m}$ and $d_{31, p}$ are the longitudinal piezomagnetic and transverse piezoelectric coefficients, respectively. Although the magnetostriction of Metglas SA1 was only 42 ppm [Fig. 2(b)], which is far smaller than the giant magnetostriction of Terfenol-D, the maximum value of its effective piezomagnetic coefficient $\left[d_{33, m}=4 \times 10^{-6} / \mathrm{Oe}\right.$, see right-hand axis of Fig. $\left.2(\mathrm{~b})\right]$ is three to four times larger than that of Terfenol-D $\left(d_{33, m}=1.2\right.$ $\times 10^{-6} /$ Oe, see Ref. 22) due to the small saturation field. This extremely low dc bias requirement is an important advantage of Metglas/PVDF laminates over other previously

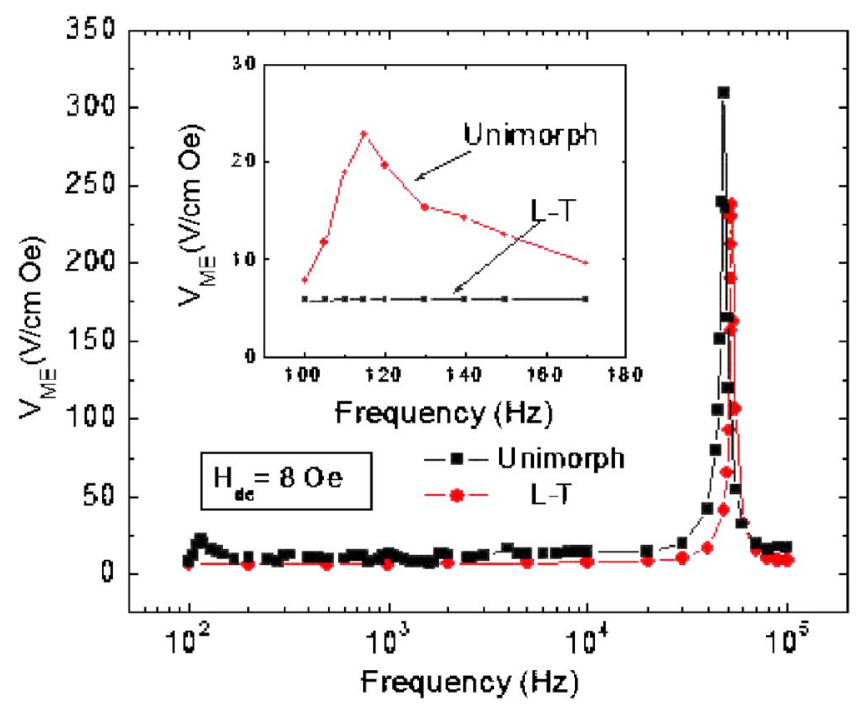

FIG. 3. (Color online) Frequency dependence $\left(10^{2}<f<10^{5} \mathrm{~Hz}\right)$ of the ME voltage coefficient of both three-layer Metgals(SA1)/PVDF and unimoprh Metglas(CO)/PVDF laminates measured under $H_{\mathrm{dc}}=8$ Oe and $H_{\mathrm{ac}}=1$ Oe. The inset shows the ME voltage coefficient of both laminate types at low frequencies, illustrating a bending-mode enhancement in the unimorph at $110 \mathrm{~Hz}$.

reported types, offering potential in practical applications. In addition, a large phase shift from $0^{\circ}$ to $180^{\circ}$ was found under small dc bias changes on the order of $1 \mathrm{Oe}$, as shown on the right-hand axis of Fig. 2(a), further offering ability to read the sign of a small moment or spin.

The three-layer sandwich laminate of Fig. 1(b) has a symmetric structure. Under a $H_{\text {ac }}$ applied along the length axis, the Metglas layers will elongate and shrink along that direction. This will force the thin PVDF layers to undergo an ac longitudinal strain, inducing a dielectric polarization change in its thickness or transverse direction. As can be seen in Fig. 3, $V_{\mathrm{ME}}$ for the three-layer sandwich laminate was flat with frequency over the bandwidth of the subresonant range, experiencing a dramatic resonance enhancement at the first longitudinal mode $f=50 \mathrm{kHz}$, with a peak value of $V_{\mathrm{ME}}=238 \mathrm{~V} / \mathrm{cm}$ Oe.

However, the two-layer unimorph laminate of Fig. 1(c) has an unsymmetrical structure. Figure 3 also shows $V_{\mathrm{ME}}$ as a function of frequency for this unimorph with $H_{\mathrm{ac}}=1 \mathrm{Oe}$ applied along the length of the sample. In addition to a principle longitudinal mode resonance near $50 \mathrm{kHz}$, a very low bending-mode resonance frequency was found. The inset of Fig. 3 shows a low frequency $(\sim 110 \mathrm{~Hz})$ resonance with a maximum $V_{\mathrm{ME}}$ of $25 \mathrm{~V} / \mathrm{cm}$ Oe (resonant-bending enhancement of approximately five times). Such low-frequency enhancement in $V_{\mathrm{ME}}$ was not observed for the three-layer structure: although, both laminate types were found to have a strong ME enhancement (three-layer, $238 \mathrm{~V} / \mathrm{cm}$ Oe; unimorph, $310 \mathrm{~V} / \mathrm{cm} \mathrm{Oe}$ ) near $50 \mathrm{kHz}$ at the longitudinal resonance frequency.

Next, we determined the sensitivity of our three-layer Metglas/PVDF laminates to small variations in ac and dc magnetic fields. Figure 4(a) shows the voltage induced by steplike changes in magnetic bias of $\Delta H_{\mathrm{dc}}=8 \mathrm{nT}$, measured in a time-domain capture mode. These measurements were performed in magnetically shielded environment under a resonant frequency $(50 \mathrm{kHz})$ drive of $H_{\mathrm{ac}}=1$ Oe: no $H_{\mathrm{dc}}$ was applied by permanent magnets. Inspection of the data will 

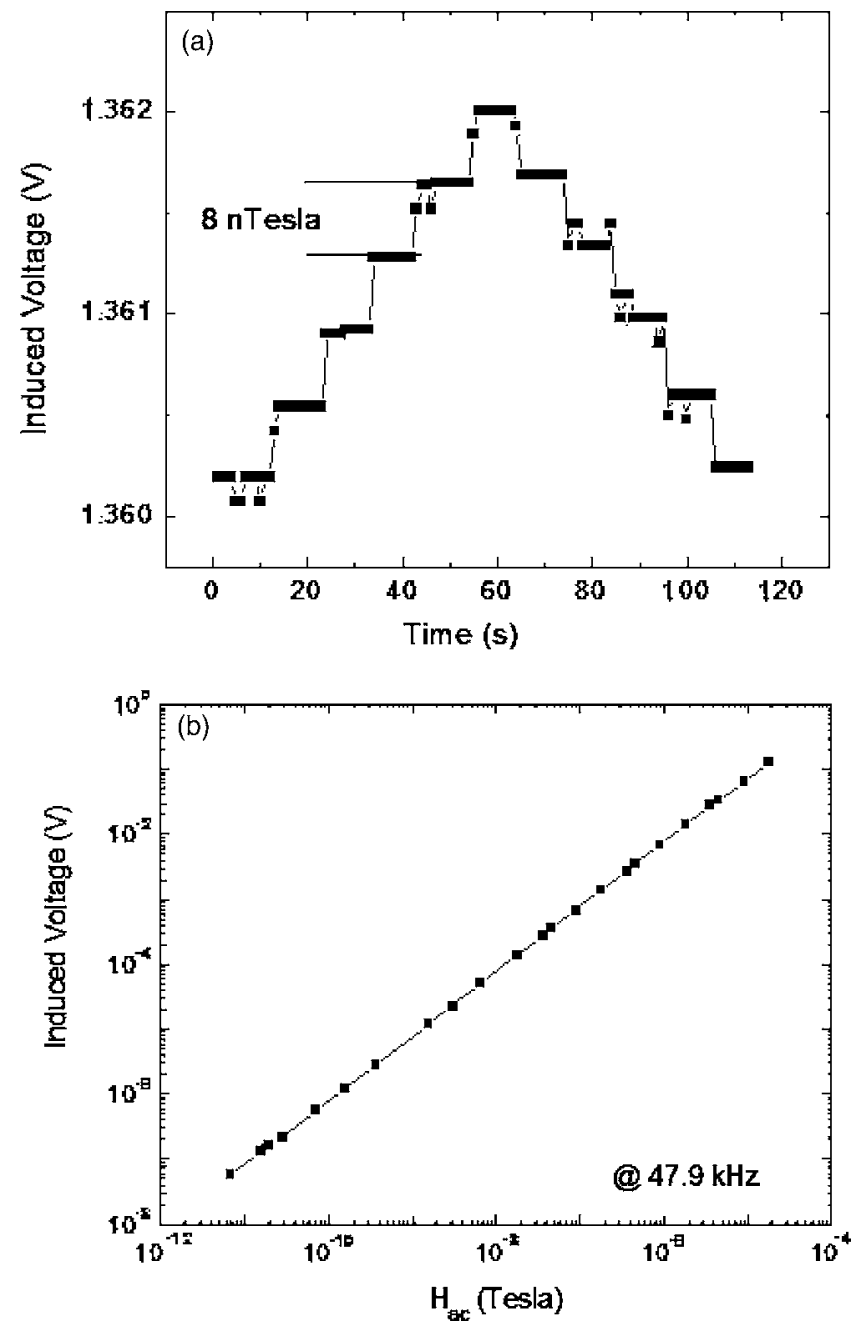

FIG. 4. Sensitivity of a three-layer Metglas(SA1)/PVDF laminate: (a) to small variations in $H_{\mathrm{dc}}$, taken under a constant $H_{\mathrm{ac}}=0.1 \mathrm{Oe}$ at $f=50 \mathrm{kHz}$, and (b) to small variations in $H_{\mathrm{ac}}(50 \mathrm{kHz})$, taken under a constant $H_{\mathrm{dc}}$ $=8$ Oe.

reveal rapid and stable responses in the voltage induced across the piezopolymer layers in response to $\Delta H_{\mathrm{dc}}$. We, then, established that steplike changes in $\Delta H_{\mathrm{dc}}$ as small as $2 \mathrm{nT}$ could be discriminated from the background noise. Finally, Fig. 4(b) shows the response of our three-layer laminate to variations in $H_{\mathrm{ac}}$ under a constant $H_{\mathrm{dc}}=8 \mathrm{Oe}$. Inspection of this figure will reveal a sensitivity limit of $<10^{-11} \mathrm{~T}$ to small variations in $H_{\text {ac }}$ over a narrow bandwidth range centered at $50 \mathrm{kHz}$ near the first longitudinal mode.
In summary, very thin, flexible, and inexpensive ME laminates consisting of Metglas/PVDF unimorph and threelayer sandwich configurations have been studied. These laminates required an applied magnetic bias of only $H_{\mathrm{dc}}$ $=8$ Oe $(1 / 50$ th that of other ME laminates $)$ to achieve a maximum ME coefficient. These small ME laminates have giant ME voltage coefficients and excellent sensitivity to small variations in both ac and dc magnetic fields.

The authors gratefully acknowledge full supported by the Office of Naval Research.

${ }^{1}$ D. N. Astrov, Sov. Phys. JETP 13, 729 (1961).

${ }^{2}$ G. T. Rado and V. J. Folen, Phys. Rev. Lett. 7, 310 (1961).

${ }^{3}$ T. Kimura, T. Goto, H. Shintani, K. Ishizaka, T. Arima, and Y. Tokura, Nature (London) 426, 55 (2003).

${ }^{4}$ M. Fiebig, J. Phys. D 28, R123 (2005).

${ }^{5}$ T. Lottermoser, T. Lonkai, U. Amann, D. Hohlwein, J. Ihringer, and M. Fiebig, Nature (London) 430, 541 (2004).

${ }^{6}$ J. Wang, J. B. Neaton, H. Zheng, V. Nagarajan, S. B. Ogale, B. Liu, D. Viehland, V. Vaithyanathan, D. G. Schlom, U. V. Waghmare, N. A. Spaldin, K. M. Rabe, M. Wuttig, and R. Ramesh, Science 299, 1719 (2003).

${ }^{7}$ M. I. Bichurin, D. A. Filippov, V. M. Petrov, V. M. Laletsin, N. Paddubnaya, and G. Srinivasan, Phys. Rev. B 68, 132408 (2003).

${ }^{8}$ G. Srinivasan, E. T. Rasmussen, J. Gallegos, R. Srinivasan, Y. I. Bokhan, and V. M. Laletin, Phys. Rev. B 64, 214408 (2001).

${ }^{9}$ G. Srinivasan, E. T. Rasmussen, B. J. Levin, and R. Hayes, Phys. Rev. B 65, 134402 (2002).

${ }^{10}$ J. Y. Zhai, N. Cai, Z. Shi, Y. H. Lin, and C.-W. Nan, J. Appl. Phys. 95, 5685 (2004).

${ }^{11}$ S. X. Dong, J.-F. Li, and D. Viehland, Appl. Phys. Lett. 83, 112265 (2003).

${ }^{12}$ S. X. Dong, J. Y. Zhai, N. G. Wang, F. M. Bai, J.-F. Li, D. Viehland, and T. A. Lograsso, Appl. Phys. Lett. 87, 222504 (2005).

${ }^{13}$ C.-W. Nan, L. Liu, N. Cai, J. Y. Zhai, Y. Yin, Y. H. Lin, L. J. Dong, and C. X. Xiong, Appl. Phys. Lett. 81, 3831 (2002).

${ }^{14}$ N. Cai, J. Y. Zhai, C.-W. Nan, Y. Lin, and Z. Shi, Phys. Rev. B 68, 224103 (2003).

${ }^{15}$ C.-W. Nan, M. Li, and J. H. Huang, Phys. Rev. B 63, 144415 (2001).

${ }^{16}$ K. Mori and M. Wuttig, Appl. Phys. Lett. 81, 100 (2002).

${ }^{17}$ J. Y. Zhai, Z. P. Xing, S. Dong, J. F. Li, and D. Viehland, Appl. Phys. Lett. 88, 062510 (2006).

${ }^{18}$ S. X. Dong, J.-F. Li, D. Viehland, J. R. Cheng, and L. E. Cross, Appl. Phys. Lett. 85, 16353 (2004)4

${ }^{19}$ R. Malmhall, G. Backstrom, K. V. Rao, S. M. Bhagat, M. Meichle, and M. B. Salamon, J. Appl. Phys. 49, 1727 (1978).

${ }^{20}$ S. X. Dong, J.-F. Li, and D. Viehland, IEEE Trans. Ultrason. Ferroelectr. Freq. Control 50, 1236 (2003).

${ }^{21}$ Calculated values based on Eq. (1) were several times higher than measured ones. In Refs. 11 and 20, a factor $\beta($ in $N / V)=1$ was empirically introduced for Eq. (1) adjustment.

${ }^{22}$ S. X. Dong, J. Zhai, F. Bai, J.-F. Li, and D. Viehland, Appl. Phys. Lett. 87, $062502(2005)$ 